\title{
Correction to: Astrophysical Fluids of Novae: High Resolution Pre-decay X-Ray Spectrum of V4743 Sagittarii
}

\author{
J.M. Ramírez-Velasquez
}

\section{Correction to:}

Chapter "Astrophysical Fluids of Novae: High Resolution Pre-decay X-Ray Spectrum of V4743 Sagittarii”" in:

J. Klapp et al. (eds.), Recent Advances in Fluid Dynamics with Environmental Applications, Environmental

Science and Engineering, https://doi.org/10.1007/978-3-319-27965-7_27

The original version of this chapter, containing text, tables, and figures, was published without the authorization of the actual author, Dr. Jan-Uwe Ness (ORCID 0000-0003-0440-7193).

Jan-Uwe Ness gives his kind permission to publish the following content retrospectively:

- Text of Sections 1, 2 (except for Subsection 2.4.1), 6, and 7.

- Tables 1, 3, and 4 .

- Figures 1, 2, 8, and 9.

Jan-Uwe Ness has now been included as Section Author in the mentioned sections. The permissions have been included in the captions of the mentioned tables and figures.

The corrected chapter has been updated with the changes. 